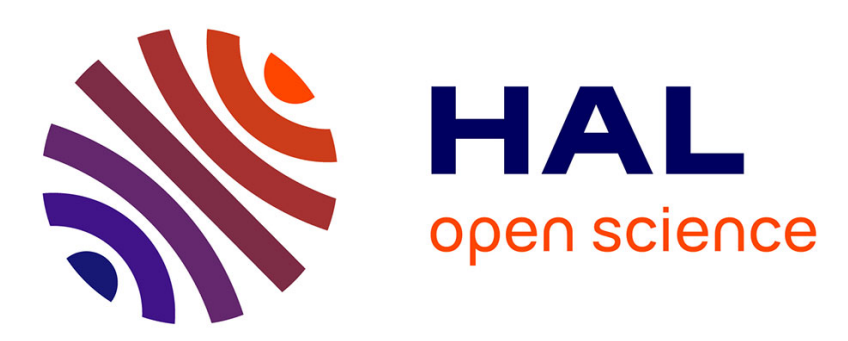

\title{
Degraded situation awareness in a robotic workspace: accident report analysis
}

Benjamin Camblor, Jean-Marc Salotti, Charles Fage, David Daney

\section{To cite this version:}

Benjamin Camblor, Jean-Marc Salotti, Charles Fage, David Daney. Degraded situation awareness in a robotic workspace: accident report analysis. Theoretical Issues in Ergonomics Science, 2021, 10.1080/1463922X.2021.1879308 . hal-03106246

\section{HAL Id: hal-03106246 \\ https://hal.inria.fr/hal-03106246}

Submitted on 11 Jan 2021

HAL is a multi-disciplinary open access archive for the deposit and dissemination of scientific research documents, whether they are published or not. The documents may come from teaching and research institutions in France or abroad, or from public or private research centers.
L'archive ouverte pluridisciplinaire HAL, est destinée au dépôt et à la diffusion de documents scientifiques de niveau recherche, publiés ou non, émanant des établissements d'enseignement et de recherche français ou étrangers, des laboratoires publics ou privés. 


\title{
Degraded situation awareness in a robotic workspace: accident report analysis
}

\author{
Benjamin Camblor ${ }^{\mathrm{a}, \mathrm{b}}$, Jean-Marc Salotti ${ }^{\mathrm{a}, \mathrm{b}}$, Charles Fage ${ }^{\mathrm{a}}$ and David Daney ${ }^{\mathrm{a}}$ \\ anria, IMS, UMR 5218, F-33400, Talence, France; b Univ. Bordeaux, CNRS, Bordeaux INP, \\ IMS, UMR 5218, F-33400, Talence, France;
}

\begin{abstract}
We adopted an approach based on interactions in a robotic workspace, coupled with Endsley's model of Situation Awareness (SA), to identify factors that potentially degrade operators' SA: the eight SA demons (Endsley and Jones 2016). We propose here to study the implications of these demons in 45 industrial accidents involving robotic. These demons were associated with possible interactions a human may have in a robotic workspace. Our results showed that five of the eight SA demons (Endsley and Jones 2016) appeared in the accident reports examined. The results showed that the identified SA demons were mainly associated with the first level of SA (perception level). We have also been able to describe five patterns of SA demon occurence in these accidents. SA demons are generally involved in human-robot interactions rather than human-environment interactions. Thanks to this analysis, we found that an operator's SA is mainly impacted by the Errant Mental Model and Out-of-the-Loop SA demons in human-robot and human-environment interactions. Finally, we propose recommendations regarding workspace design.
\end{abstract}

\section{KEYWORDS}

Industrial robotics $\cdot$ accidents $\cdot$ situation awareness $\cdot$ interaction $\cdot$ accident analysis

\section{Introduction}

Despite the plethora of norms and safety procedures that have been implemented in industry to integrate robotic systems, accidents are still reported and are often caused by human error. Robots not only allow loads to be moved using several degrees of freedom, but also exert forces, sometimes considerable, on items in the process (ISO 2012). In industrial automation applications, an automatic machine becomes a robot, depending on the complexity of the generated movement, its adaptation to the environment and its degree of autonomy. Because robotic movements may be dangerous, robots are usually placed in robotic cells with stringent access restrictions. However, robots are rarely isolated machines. The means of perception, gripping and other production elements, such as a conveyor, a press, or a packaging machine, etc., are in general closely linked to the activity of the robot and are located in the same workspace. As human errors and robotic accidents may involve different elements of the robotic workspace, we propose to define a robotic system as the set of all elements of the robotic environment that are involved in the task performed by the robot.

There has been an increase in the number of accidents in line with the increase in the number of robots in the industrial arena (Dhillon 1991). Previous studies have investigated the reasons for such accidents. Human error (Jiang and Gainer Jr 1987), 
incorrect decision making by operators (Sugimoto and Kawaguchi 1983; Jiang and Gainer Jr 1987; Malm et al. 2010) and hazards associated with workspaces (Sugimoto and Kawaguchi 1983; Or, Duffy, and Cheung 2009; Malm et al. 2010; Charpentier and Sghaier 2012; Vasic and Billard 2013) are among the most cited reasons. Considering these findings, an interest in situation awareness in accidents involving industrial robotics has emerged (Salotti and Suhir 2019). Situation Awareness (SA) is the perception of the elements in the environment within a volume of time and space, the comprehension of their meaning, and the projection of their status in the near future (Endsley 1995). Indeed, it seems that those hazardous situations in which the operator made a wrong decision are due to a degraded SA. Eight factors impacting situation awareness - named SA demons (Endsley and Jones 2016) - are potentially involved in industrial accidents concerning robotics (Salotti and Suhir 2019).

In this vein, this paper presents a new method for analysing robotic accidents in industrial environments in order to better understand the human factors involved and to make recommendations to reduce the risks. It is based on Endsley's Situation Awareness model, which will be presented in Section 2 and the analysis of a database of robotic accidents that occurred in various industrial contexts. Our general objective is to provide solutions to maintain SA within an industrial robotics workspace and thus limit the risk of accidents. In this context we aim to find new solutions or signals that a human and a robot can share to give each other information about their current state. Particularly, we will focus on information that a robot can share with a human being to maintain the latter's SA. It is necessary to find which factors can negatively impact an operator's SA. To do this, we will first analyse robot-related industrial accidents using assessment criteria based on SA demons and interactions in the robot workspace. This analysis will allow us to pinpoint the critical factors involved, in order to develop novel and relevant solutions to prevent such accidents in the future.

Here we propose to investigate accidents involving industrial robots recorded in the French database EPICEA (INRS). In section 2, the state of the art concerning hazardous human-robot interactions, human error \& SA and accident analysis is presented. In section 3, the method we used for accident analysis is explained. Using the keyword robot 55 accidents have been identified within industrial workplaces. For each accident, we identified SA demons and associated them with interactions between a human and the other elements of the robot workspace. Thus, we identified factors based on SA that led to an accident. Our results are presented in section 4. Five SA demons have been identified and associated with interactions in the robot workspace. Patterns of SA demon occurrence have also been found. In section 5, these results are discussed in the light of our objective of preventing industrial robots accidents. Finally, the conclusions of this work and recommendations regarding robot workspace design are presented in section 6 .

\section{Related Work}

According to Dhillon, an industrial robot is defined as an automatically controlled, reprogrammable multipurpose manipulator (Dhillon 1991). It can be programmable in three or more axes and can be either fixed in place or mobile (ISO 2012). A robotic system has been defined as a system including human beings and a communication system (Jiang and Gainer Jr 1987). Next, a taxonomy has been suggested in which the human-robot interaction can be formally defined as a quintuple: (i) task requirements, (ii) user characteristics, (iii) robot characteristics, (iv) environment and (v) a set of 
interactions (Rahimi and Karwowski 1990). This is close to the definition of a collaborative workspace where a robot system and a human being can concurrently perform tasks during a production operation within an operating space (ISO 2011b,a, 2016). Additionally, a framework including a human being and a robot interacting thanks to an interface in an environment has been proposed to model interactions in a robotic context (Salotti et al. 2018). We consider the concept of the robot workspace as the environment where a human being and a robot are interacting to perform a task.

\subsection{Hazardous Human-Robot Interaction}

On one hand, conventional industrial robots are fixed installations that can repeat a defined task. In general, operators are not supposed to be get close to them and they are almost invariably separated from humans by physical barriers and the robotic cell is equipped with sensors to avoid physical human/robot interactions (Villani et al. 2018). On the other hand, thanks to collaborative robot (Colgate et al. 1996) - cobots -, industrial robotic development is moving from "robot in cage" to "robot near human". They can easily be moved and are light in weight (Villani et al. 2018). Conversely, cobots are intended to be in direct interaction with the operator. In this way, physical interactions between humans and robots are increasingly desirable and essential. Such interactions must take place under safe and operator-friendly conditions (Ogorodnikova 2008).

Therefore, safety is the inherent and most important feature of a robot that has to work in close cooperation with human beings (De Luca and Flacco 2012). There are almost thirty active EU directives and around six hundred different standards related to safety (Michalos et al. 2015). Security norms aim to prevent human access to the robot workspace or to stop the robot when an unexpected event in the workspace is detected by external sensors (Meziane et al. 2014). Nevertheless, accidents still occur when a person needs to perform a task alongside a robot (Malm et al. 2010). The use of the current safety measures can be challenging when a person and a robot are in close proximity. Indeed, regarding conventional robots, whilst getting close to the robot zone is prohibited, operators of all types still spend around three hours a day working close to them (Aghazadeh, Hirschfeld, and Chapleski 1993). Even if interactions are not desired, operators need to perform maintenance, cleaning, troubleshooting, repairing and 'teaching' the robot. They may also work near an operational robot or a robotic workcell. All of these reasons lead to hazardous situations in the robot workspace.

Hazardous conditions are mainly related to intrusion by human operators into a robot workspace in either operational or non-operational phases (Sugimoto and Kawaguchi 1983). Thus the close physical interaction between human beings and robots may result in a high degree of risk of an accident (Karwowski and Rahimi 1991). Unnatural and unexpected movements may be responsible for $73 \%$ of robotrelated injuries and fatalities (Sugimoto and Kawaguchi 1983). The interpretation of a robot stoppage is one of the other main hazard categories. This results in a perceptual problem for the operator and incomprehension of the robot state. This would lead to a degraded situation awareness for the operator.

\subsection{Human error and situation awareness}

Various methods or tools are used for analysing human activities and human factors involved in accidents. They focus on the level of knowledge of the operator (SRK (Rasmussen 1983)), or on active and latent potential errors causing accidents (Reason 
(Reason 1990), HFACS (Shappell et al. 2007), European Assembly Worksheet (Schaub et al. 2013)). However, these broad methods fail to capture the cognitive aspects of human error in accident analysis. The Endsley model of Situation Awareness can be leveraged to achieve this goal.

According to Endsley, situation awareness can be divided into three levels (Endsley 1988, 1995). The first level (SA level 1) is related to the perception of the elements present in the environment. In industrial robotic applications, the operator perceives first the global state of all elements of the robotic system (e.g. the position and moves of the robot, the state of alarms, etc.). In general, the perception level also includes a command panel that is located outside the cell and is used to start and stop the robot and other elements of the cell. The second level (SA level 2) is related to the comprehension of the current situation. Based upon his/her knowledge of the perceived elements, the operator forms a holistic picture of the environment and can link the behaviour of the robot and the state of each element of the cell to a normal or an abnormal situation. In industrial applications, the current situation is sometimes complex and an abnormal robot behaviour (e.g., the robot stops) may be linked to a problem with other machines or sensors, a misunderstanding of a starting or maintenance procedure, the action of other operators, etc. The third level (SA level 3) is about the ability to make a projection of the future state, determining the consequence of subsequent actions and the evolution of all elements of the environment, including the various parts of the robotic system. According to Suhir et al. (2015), the major components of that projection are environment awareness - here the workspace -, system awareness - here the robot -, and task awareness. SA plays a major role in the process of decision making (Endsley 1995). If a decision is made in the context of degraded situation awareness, there is a higher probability of human error, which could be the main cause of an accident. Additionally, various mechanisms or factors may be at work to modify or alter one or more levels of SA (Endsley and Jones 2016). These are called "situation awareness demons" and are classified into eight categories. Each SA demon may concern one or more specific SA level among perception, comprehension and projection (Endsley and Jones 2016; Stratmann and Boll 2016). It is proposed to add the industrial robotics context to their proposed definitions, as follows:

- Attentional Tunneling - AT (SA level 1): Good SA is dependent on switching attention between multiple data streams. Locking in on certain data sources and excluding others is attention tunneling.

In industrial robotics: Occurs when an operator is focused on a task or on a particular feature of the environment and therefore misses relevant information from his/her environment.

- Out-of-the-Loop - OL (SA level 1 and 2): Automated systems that do not involve the operator until there is a problem (for example). It can be caused by vigilance/monitoring problems or information feedback problems (level $1 \mathrm{SA}$ ), as well as lower engagement (level $2 \mathrm{SA}$ ).

In industrial robotics: Occurs when a robot is performing its actions while the operator is performing his/her own actions regardless of what the robot is doing.

- Errant Mental Model - EMM (SA level 2 and 3): An incomplete mental model may result in a poor comprehension and projection of the situation. Additionally, a wrong mental model may be used to interpret the situation.

In industrial robotics: Occurs when an operator misinterprets the situation and then makes inappropriate inferences about the state of the environment.

- Complexity Creep - CC (SA level 1, 2 and 3): Complexity slows down the per- 
ception of information and it undermines the understanding and the projection of information.

In industrial robotics: Occurs when a problem is encountered and there are so many systems involved that the operator is not able to isolate the problem in such a complex situation and find a solution.

- Misplaced Salience - MS (SA level 1): Salience is the "compellingness" of a piece of data, often dependent on how it is presented.

In industrial robotics: Occurs when the system is designed to maximise the operator's concentration on a specific device or event and therefore impedes his/her access to more relevant information regarding the situation.

- Data Overload - DO (SA level 1): If there is more data available than can be processed by the human "bandwidth".

In industrial robotics: Occurs when too many data elements have to be set or taken into account in a context involving important attentional or time constraints.

- Requisite Memory Trap - RMT (SA level 1 and 2): The working memory processes and holds chunks of data to support SA level of comprehension. Systems that rely on robust memory do not support the user.

In industrial robotics: Occurs when too many subtasks have to be performed and the operator forgets one of them.

- Workload, Anxiety, Fatigue, and Other Stressors - WAFOS (SA level 1,2 and 3): Stress and anxiety are likely issues in the warning environment. WAFOS taxes attention and working memory.

In industrial robotics: Significant "workload, fatigue, or stress" has obviously a direct impact on performance and may be a contributor to human error.

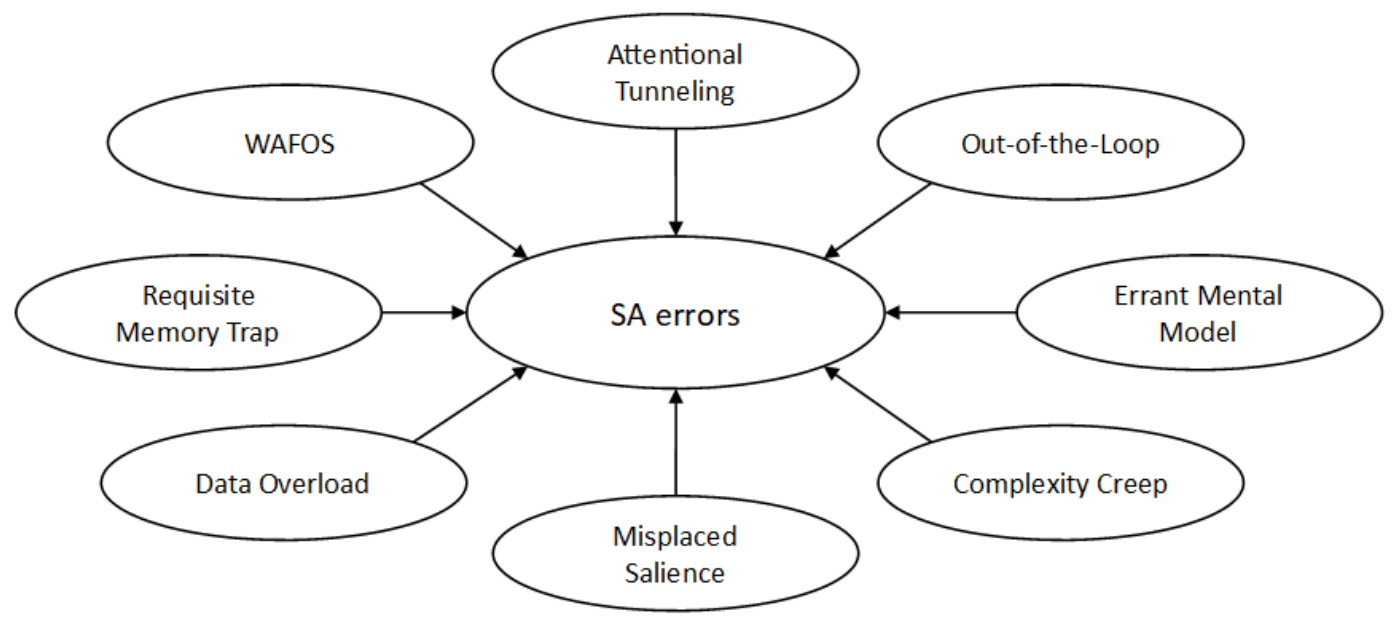

Figure 1. SA demons which can cause an SA error (Salotti and Suhir 2019)

\subsection{Situation Awareness and Accident analysis}

To the best of our knowledge, accident analysis and situation awareness have not yet been linked together in the field of industrial robotics, whereas they have already been highlighted in both the aviation (Jones and Endsley 1996; Endsley and Jones 2016) 
and maritime domains (Stratmann and Boll 2016). First, linking SA and human error analysis in aviation accidents highlighted that $76.3 \%$ of errors were classified as "level 1 SA errors" $1,20.3 \%$ were "level 2" 2 and $3.4 \%$ were "level 3" 3 (Jones and Endsley 1996). Moreover, in the aviation world, "SA demons" have even been specified as a requirement for analysing aviation accidents (Endsley and Jones 2016; Salotti and Suhir 2019).

In the maritime domain, researchers have shown that an accident can involve more than one SA demon and that "SA level 1" degradation appears to be the most prominent cause of human error (Stratmann and Boll 2016). They also studied the proportion of SA demon appearances in accidents whenever this was possible. As for accidents in industrial robotics, their main analysis focused on human error and workplace design (Jiang and Gainer Jr 1987; Malm et al. 2010), the misunderstanding of the robot's state (Or, Duffy, and Cheung 2009) and the presence of operators in the robot workspace (Aghazadeh, Hirschfeld, and Chapleski 1993; Charpentier and Sghaier 2012; Lamy and Tissot 2016). More specifically, Charpentier and Sghaier (2012) and Lamy and Tissot (2016) have analysed accidents from EPICEA (INRS), a French industrial accident database. It has been demonstrated that a significant proportion of accidents occurs during the operational phase (i.e. when the robot is performing a task) (Charpentier and Sghaier 2012). Finally, Salotti recently suggested the potential involvement of SA demons in numerous industrial robotics situations (Salotti and Suhir 2019). Consequently, it is proposed here to analyse these accidents and identify the SA demon manifestation in such cases.

\section{Method}

\subsection{EPICEA database}

This study analysed industrial accidents involving robots in the light of the SA demons presented in the previous section. Accident reports were extracted from the EPICEA ${ }^{4}$ database. This is an anonymous national French database provided by the French Institute of Research and Security. Although this database is not exhaustive, some 24,000 work accidents have been recorded since 1990 in French companies (Lamy and Tissot 2016). The recorded accidents are labelled with different levels of consequences, which can be either fatal, serious (e.g. the operator has been injured) or significant (e.g. some working equipment has been damaged). The purpose of the EPICEA database is to highlight the potential causes and development of accidents, without seeking to establish responsibility, and to provide illustrative cases for awareness-raising, training, etc.

\subsection{Analysis}

All accident reports are labelled with keywords. In order to focus on robotic accidents, the decision was made to use the keyword robot. Using this keyword 55 accident reports were collected. Other keywords were tried ("robotique" for example) but did not produce any result. All reports were systematically reviewed. Accident reports have

\footnotetext{
${ }^{1}$ Perception

${ }^{2}$ Comprehension

${ }^{3}$ Projection

${ }^{4}$ Etudes de Prévention par l'Informatisation des Comptes-rendus d'Enquêtes d'Accidents
} 
been analysed by three raters. The analysis was conducted independently by the three raters before joining together the results. Raters agreed for 12 of the 55 accidents. For the 43 remaining reports, each one has been discussed and agreed. Some accident reports were rejected because they lacked important information for understanding what really happened $(\mathrm{N}=2)$. In some cases, there was a robot in the accident environment but the incident did not directly result from an issue with the robot or an interaction with it $(\mathrm{N}=8)$. For instance, a robot and a press are located in the same workspace and the robot is not used. There is a problem with the press, the operator enters the robotic cell and is injured because the press was not stopped. This type of accident was rejected. This review resulted in a pool of 45 accident reports involving robotic systems.

For each of the 45 accident reports, an analysis of the task was carried out. First, as is usual in the field of ergonomics, the accident is split into phases highlighting the detailed sequence of events. Secondly, using SA demons as an assessment criteria, the manifestation of SA demons was highlighted during the various phases. Every SA demon raised has been debated between raters. At the end, the SA demons chosen were the result of an agreement between the raters. And thirdly, once the demons were identified, interactions were analysed and categorised into "human/robot", "human/environment" or "human/task". Once again, the raters agreed on the associated interactions. An example is provided in the following sections.

\subsection{Demon identification: Decision Making}

As SA demons have not been extensively used to analyse industrial robotic accidents, this paragraph provides complementary elements to help identifying their presence. These elements are meant as guidelines for SA demon identification.

- Attention Tunneling - AT: Is identified when the operator is focused on his/her task while in the robot workspace, regardless of the robot's actions.

- Out-of-the-Loop - OL: Is identified when an operator missed an important event and a normal but unexpected action of the robot is observed.

- Errant Mental Model - EMM: Is identified when the operator has - or seems to have - used an erroneous inference rule to determine the state of the robot and its environment. For instance, deciding to enter the robotic cell while the robot is not moving, thinking that it is stopped without effectively checking the robot status.

- Complexity Creep - CC: Is identified when there are too many procedures for the use or the maintenance of the robot or when the system or the interface is too complex for the operator.

- Misplaced Salience - MS: Is identified when an element of the interface (typically the safe-guarding system) is not clearly indicating the relevant information to the operator, or can mistakenly be confused with another.

- Data Overload - DO: Is identified when the environment, the robot or the command panel provide too much information for the operator's capacity.

- Requisite Memory Trap - RMT: Is identified when the operator forgets an important step in the task sequence.

- Workload, Anxiety, Fatigue and Other Stressors - WAFOS: Accident reports are often lacking information about the psychological state of the operator. Nevertheless, it was sometimes mentioned that the operator was tired or stressed, which is thus an explicit and useful piece of information. 
These elements helped us identifying the presence or absence of each of the $8 \mathrm{SA}$ demons for each of the 45 robotic accidents. Several demons may be identified in the same accident report. Different relationships exist among them. It can be a temporal sequence, a causality bond or a simple co-occurrence. Sometimes, the same set of demons with the same relationship is identified in several accident reports. This peculiarity will be discussed later. Finally, if the same SA demon is identified for different phases of the accident, it is considered only once for statistical purposes.

\subsection{Example}

Description of the accident:"The victim, a 27-year-old press operator, was in the injection moulding machine workshop. While he/she was working, he/she stood up to pick up pieces from the other side of the conveyor. In order to do so, he/she had to move into the area of the evacuation manipulator robot. At that moment the robot that was performing its descent hit the victim near the scapula. The shock caused various contusions requiring hospitalisation."

\subsubsection{Phase splitting}

First, the accident was split into different phases according to the chronological order of events.

S1. The victim enters the injection moulding machine workshop.

S2. He/She moves into the area of the evacuation manipulator robot.

S3. He/She stands up to pick up pieces from the other side of the conveyor.

S4. The robotic arm moves and injures the victim.

\subsubsection{Analysis with $S A$ demons}

Following the decomposition into phases, SA demons have been associated with each phase whenever possible.

S1. According to available information, no demon during this phase.

S2. The victim should not be present in the robot workspace while the robot is switched on. He/She is not following the safety procedure, as he/she had in mind a wrong interpretation - errant mental model - of the situation.

S3. He/She is manipulating pieces while not paying attention to the robot position. During this phase, the victim seems to be in an attentional tunneling state.

S4. Finally, because he/she is in an attentional tunneling situation, the victim does not perceive that the robot is getting dangerously close to him/her. There is an out-of-the-loop situation.

\subsection{3. $S A$ demons and interactions in the robot workspace}

Finally, SA demons can be associated with an interaction with the operator in the robot workspace.

S1. No SA demons identified so we could not associate an interaction.

S2. Errant Mental Model: The victim is entering a potentially dangerous environment where a robot is operational. The SA demon can therefore be assigned to the human-environment interaction group. Also, because the robot is the source of hazard, it can also be assigned to the human-robot interaction group. 
S3. Attentional Tunneling: As the operator is focusing on his/her task, the SA demon can be assigned to the human-task interaction group.

S4. Out-of-the-Loop: As the operator is out-of-the-loop of the robotic sequence of actions, the SA demon can be assigned to the human-robot interaction group.

\section{Results}

The systematic analysis of the EPICEA database allowed us to identify the SA demons participating in degrading the operators' SA and potentially leading to the accident itself. We were also able to identify recurrent patterns of SA demon appearances, enabling categorising the accidents. Finally, a further analysis based on interactions is presented, providing insights into SA demon appearances across elements of the robotic cell.

\subsection{SA demon manifestation}

\subsubsection{SA demon occurrences}

Regarding the occurrences of SA demons across the robotic accidents in the EPICEA database, three main results can be highlighted (see Table 1).

Table 1. Number of SA demon occurrences for each accident in the database.

EMM: Errant Mental Model ; OL: Out-of-the-Loop; AT: Attentional Tunneling; CC: Complexity Creep; MS: Misplaced Salience; DO: Data Overload; RMT: Requisite Memory Trap; WAFOS: Workload, Anxiety, Fatigue and Other Stressors.

Examples: EMM: wrong belief robot is off - OL: misunderstanding why robot stopped - AT: focusing on a task - MS: confusing alarm system - CC: complex procedure - DO: excessive amount of information to process RMT: forgetting a step in the procedure - WAFOS: any physiological degradation.

\begin{tabular}{l|llll}
\hline SA demons & EMM & OL & AT & MS \\
\hline $\mathrm{N}$ & 45 & 39 & 18 & 14 \\
Proportion & $100 \%$ & $86.67 \%$ & $40 \%$ & $31.11 \%$ \\
\hline SA demons & WAFOS & CC & DO & RMT \\
\hline $\mathrm{N}$ & 1 & 0 & 0 & 0 \\
Proportion & $2.22 \%$ & $0 \%$ & $0 \%$ & $0 \%$ \\
\hline
\end{tabular}

First, the most common SA demons encountered were the Errant Mental Model demon and the Out-of-the-Loop demon, with $100 \%(\mathrm{~N}=45)$ and $86.67 \%(\mathrm{~N}=39)$ of appearances respectively. Errant Mental Model appears in all of our accidents. It occurs when the operator misinterprets the situation and then makes inappropriate inferences about the state of the environment, such as thinking that the working area of the robot is safe while the robot is still switched on. In addition, an operator who enters the robot the area without knowing that it is turned on would already be in an Out-ofthe-Loop situation. This latter occurred when the operator was not prepared to the restart of the robot's activity. Moreover, we report two demons appearing relatively frequently. The Attentional Tunneling $(\mathrm{N}=18)$ and the Misplaced Salience $(\mathrm{N}=14)$ respectively appear in $40 \%(\mathrm{~N}=18)$ and $31.11 \%(\mathrm{~N}=14)$ of reported robotic accidents. In addition, three demons were never identified across robotic accidents. Indeed, we have not accounted for any manifestation of Complexity Creep, Data Overload and 
Requisite Memory Trap. This might be due to the lack of detail in the reports. Finally, WAFOS was identified in only one accident - the only one where stress was explicitly reported.

\subsubsection{SA levels in industrial accidents involving robotics}

Once the SA demons were identified, we grouped them by SA level. We used the classification proposed by Stratmann and Boll (2016). In the context of SA levels, the SA demons we identified relate more to the first level (Perception) in $44.17 \%$ ( $\mathrm{N}=72$ ) of cases. They relate, to a lesser extent, to the second level (Comprehension) for $28.22 \%$ $(\mathrm{N}=46)$ of cases and the third level (Prediction) for $27.61 \%(\mathrm{~N}=45)$ of cases.

Specifically, the Perception level of SA groups 4 demons: Out of the Loop (33.33\%), Attentional Tunneling (15.38\%), Misplaced Salience (11.97\%) and WAFOS (0.85\%). The comprehension level captures only 2 demons: Errant Mental Model (38.46\%) and WAFOS $(0.85 \%)$. Note that WAFOS only appears once for levels Perception and Comprehension. Finally, only the Errant Mental Model (38.46\%) appears within the Prediction level of SA.

\subsubsection{Patterns of $S A$ demons in these accidents}

Five accident patterns were also identified through this analysis where it is possible to observe identical occurrences of SA demons leading to any one of these accidents. These five patterns are represented in Figure 2.

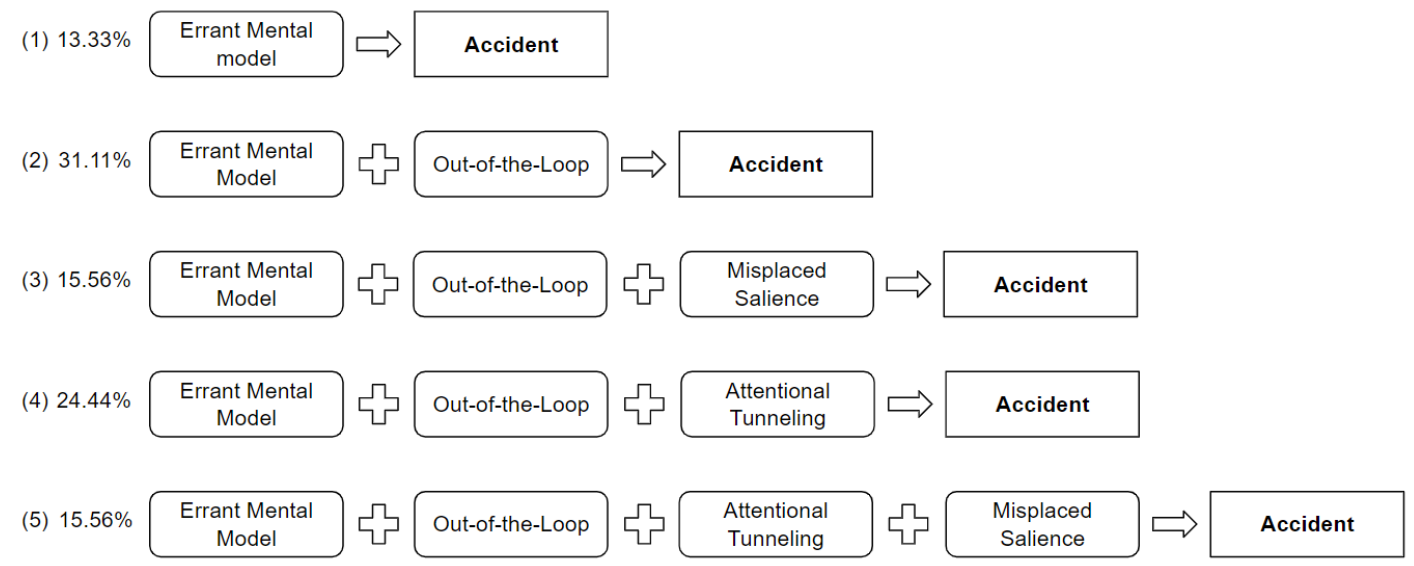

Figure 2. SA demon sequence patterns deduced from our analysis of industrial accidents involving robotics. (1) EMM leading to an accident - $13.33 \%$ of cases. (2) EMM and OL leading to an accident - $31.11 \%$ of cases.

(3) EMM, OL and MS leading to an accident - $15.56 \%$ of cases. (4) EMM, OL and AT leading to an accident - $24.44 \%$ of cases. (5) EMM, OL, AT and MS leading to an accident $-15.56 \%$ of cases.

If we focus on each of the patterns, the first one appears in $13.33 \%$ of cases $(\mathrm{N}=6)$. Example: an operator entering the robot area while the robot is still on and thinking it is safe. The second occurs in $31.11 \%$ of cases $(\mathrm{N}=14)$. Example: an operator entering the robot area while it is switched on (EMM) and being hit after a sudden move of the robot $(\mathrm{OL})$. The third appears in $15.56 \%$ of cases $(\mathrm{N}=7)$. Example: an operator entering the robot area while it is switched on (EMM), he/she thinks that he/she turned it off (MS), and he/she is hit after a sudden move of the robot (OL). The fourth occurs in $24.44 \%$ of cases $(\mathrm{N}=11)$. Example: an operator entering the robot 
area while it is switched on (EMM) and is hit by the robot (OL) while being focused on a particular task (AT). Finally, the last pattern appears in $15.56 \%$ of cases $(\mathrm{N}=7)$ as the third one. Example: an operator entering the robot area while it is switched on (EMM), he/she thinks that he/she turned it off (MS), and he/she is hit by the robot (OL) while being focused on a particular task (AT).

We can observe that Errant Mental Model manifests itself first in all the patterns we found. Also, it alone defines one of the patterns that occurs $13.33 \%$ of the time. Additionally, the SA demon Out-of-the-Loop appears in all the other patterns (86.67\% of the cases). Specifically, it always appears after the Errant Mental Model. Finally we found SA demons Attentional Tunneling and Misplaced Salience in 2 patterns (each in $40 \%$ of the cases).

The analysis with the assessment criteria related to SA demons in accidents revealed a manifestation of Errant Mental Model in all cases. In the majority of cases, this event was related to phases in which the operator was described as "entering the area of the robot without switching it off". However, even if the presence of an operator in the robot workspace is due to an erroneous mental model, there are other SA demons that have arisen before the accident. Indeed, Errant Mental Model manifested itself with at least an Out-of-the-Loop manifestation before an accident in $86.67 \%$ of cases. In almost half of the cases, an Out-of-the-Loop occurs because the victim was focused on his/her task (Attentional Tunneling), or on another element of the environment. Finally, Misplaced Salience manifested itself when the safety devices were unable to warn the operator about a possible accident in the robot workspace. These last two demons were responsible for the appearance of the Out-of-the-Loop demon. Tunneling led the operator to focus on something other than the robot, putting him/her out of the loop. Inadequate salience did not inform the operator of the robot's status and potential next actions.

Having identified the SA demons in the accident reports, it is now possible to link them with the interactions within the robot workspace.

\subsection{Accident analysis in the robot workspace}

\subsubsection{SA demons and interaction errors}

For accidents in the robot workspace, interaction errors are mainly Human-Robot (46.29\%) and Human-Environment (42.29\%). Human-Task (11.42\%) interaction errors are found to a lesser extent and almost entirely relate to the Attentional Tunneling SA demon. Table 2 shows the number of times each of the five demons identified has been associated with each of the interactions with humans in the robot workspace.

Table 2. Number of interactions with humans in the robot workspace for each SA demon counted. HR: Human-Robot interaction, HE: Human-Environment interaction, HT: Human-Task interaction.

\begin{tabular}{l|llllll}
\hline & EMM & OL & AT & MS & WAFOS & Total \\
\hline HR & 39 & 39 & 0 & 2 & 1 & $81(46.29 \%)$ \\
HE & 45 & 14 & 0 & 14 & 1 & $74(42.29 \%)$ \\
HT & 1 & 0 & 18 & 0 & 1 & $20(11.42 \%)$ \\
\hline
\end{tabular}




\subsubsection{Classification of $S A$ demons in interaction categories}

We can now highlight the SA demon manifestations within the interactions in the robot workspace. Figure 3 shows the classification of SA demons in the robot workspace.

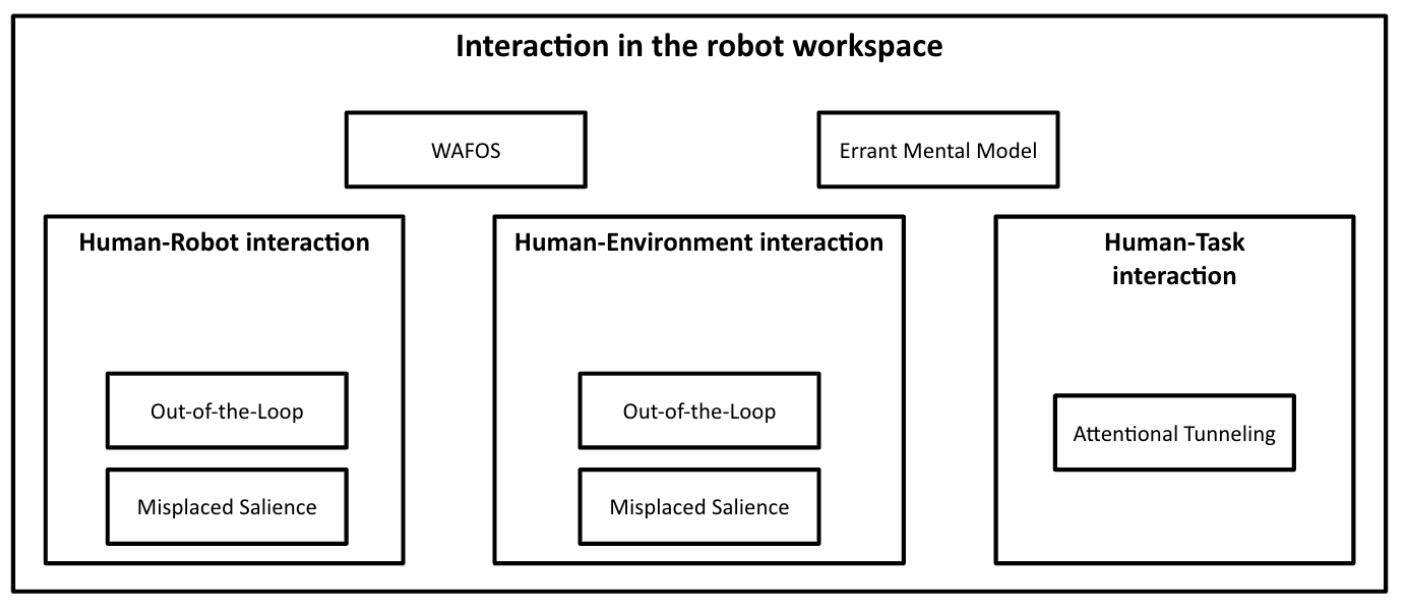

Figure 3. SA demon classification in robot workspaces according to our results.

Out-of-the-Loop and Misplaced Salience are both linked to human-robot and human-environment interactions. Attentional Tunneling is linked to human-task interaction. Finally, WAFOS and Errant Mental Model are linked to all the interactions that can happen in the robot workspace.

Each demon belongs to the smallest rectangle in which it is registered. Thus, we can highlight that the SA demon Errant Mental Model appears in all three interaction types and, more precisely, in the majority of the Human-Robot and HumanEnvironment interactions. The SA demon Out-of-the-Loop is the second most frequent demon to appear. It is only concerned human-robot and human-environment interactions. We found the SA demons Attentional Tunneling (human-task and humanenvironment) and Misplaced Salience (human-robot and human-environment) to a lesser extent. Finally, we found only one occurrence of the SA demon WAFOS and this in all three interaction types. Because Errant Mental Model and WAFOS are mainly linked to the human mental state, they appear in all three interaction types and thus belong in the robot workspace box that encompasses all interactions. Otherwise, each demon manifests itself in the interactions associated with the boxes in which it belongs.

\section{Discussion}

The aim of this work is to address the problem of industrial accidents involving robotics from a cognitive point of view in order to try to develop new safety solutions by investigating SA. We focused on information that the robot can share with an operator to maintain the latter's SA. This kind of information can be used to enhance the design of a robot workspace in the context of industrial robotics. First, the method presented in this paper brings an additional point of view to existing factors causing such accidents. Next, the perception level of SA as the main source of error is discussed. Afterwards, SA demon association and sequencing are addressed. Then SA demons and interactions in the robot workspace are linked in order to focus on improving the safety aspects of robot workspaces. Finally, the limitations of this type of accident analysis 
are discussed.

\subsection{Factors causing accidents involving robotics}

Our results showed that SA demons intervened during the events leading up to an accident, and the identification of these SA demons enabled labelling the already known main causes of accidents. Errant Mental Model appeared in all of our accident cases. Most of the time, the SA demon manifested itself because the victim intervened in the area of the robot while it was still in operation. In other cases, the operator bypassed the safety mechanisms. As accident reports are not consistently detailed, it was sometimes difficult to understand the operator's decision-making process. In such situations, we were obliged to make assumptions based on the documentation concerning the reasons that led the operators to act in the way they did. The main possible reason would be the operator's false belief that "a motionless robot is a powerless robot" (Sugimoto and Kawaguchi 1983). The underlying erroneous mental model would then be related to this false belief of feeling safe when the robot is inactive.

The Out-of-the-loop SA demon was the second most prevalent $(86.67 \%)$ in our analysis. This corresponds to a movement of the robot not anticipated by the victim. In $60 \%$ of the cases, such an occurrence is directly linked with the demon Errant Mental Model as the operator maybe thought there was no risk involved. This may correspond to the situation where the operator is not aware of the current status of the robot and thinks that it is possible to enter the work area in total safety (Or, Duffy, and Cheung 2009). In $40 \%$ of the cases, the robot was no longer perceived because the operator was strongly focused on his/her task. This is when SA demon Attentional Tunneling manifests itself ; this situation where the operator is focused on solving another problem has already been highlighted by Malm et al. (2010).

The Misplaced Salience SA demon appeared in $31 \%$ of cases. Its appearance is linked to the lack of accessible or sufficiently salient information that could help the operator understand the current situation. In most cases, its manifestation was a result of a lack, or a removal, of security safeguards. In other cases, the system warnings were said to be inadequate. Such reasons have already been pointed out as responsible for accidents (Jiang and Gainer Jr 1987).

Only one appearance of the WAFOS SA demon was noted. Accident reports do not specify the constraints that the operators may have: there is generally no information about his/her mental state. Thus, WAFOS may have been involved more than once among the cases in our analysis. As a comparison, Stratmann and Boll (2016) reported it in $40 \%$ of their maritime accident cases. Conversely, they did not find any manifestation of the Misplaced Salience SA demon of insufficient detail in their data. In addition, several works have already addressed stress or anxiety issues of operators when interacting with robots. operators sometimes feel high levels of mental strain when a robot moves near them has been highlighted in the literature (Arai, Kato, and Fujita 2010). It has also been shown that fast or unpredictable movements of a robot leads to increased cognitive load or anxiety (Koppenborg et al. 2017).

Finally, no occurrences of the SA demons Complexity Creep, Data Overload and Requisite Memory Trap were found. The same results for Complexity Creep and Requisite Memory Trap were found in the analysis by Stratmann and Boll (2016) of maritime accidents. Again, these SA demons are harder to find and heavily dependent on the accident report. Complexity Creep is said to be a "subtle SA demon" (Endsley and Jones 2016). It occurs when many machines or functionalities are involved in the sys- 
tem. Measuring the complexity of a system using the accident reports we had at our disposal was not simple. This would have required more detail about the complexity of the tasks or systems in the reports. We did not find any information about the complexity for operators. Requisite Memory Trap relies on the operator span and the way he/she can monitor his/her tasks (Endsley and Jones 2016). This makes it relatively hard to identify in an accident report because it is entirely up to the operator (Chiappe, Strybel, and Vu 2012). As in the case of WAFOS, the identification of Data Overload is directly linked to the accident report and the available information. Unfortunately, the reports do not mention the cognitive load of the operators at the time of the accident. It would have been valuable, when feasible, to be able to interview accident victims in order to get information about it.

\section{2. $S A$ demons and $S A$ levels}

With regard to SA levels, the demons identified were primarily related to the level of perception (44.17\% of cases) followed by the levels of comprehension $(28.22 \%)$ and prediction (27.61\%). These results are consistent with Stratmann and Boll (2016). However, this description is static and does not account for the chronological appearance of the SA demons. Since SA is a dynamic process, it may be that at a first turn the operator's perception of the situation is the "right" one. His/Her perception at the next turn could then be degraded because he/she has a poor understanding of the situation or he/she makes a bad prediction about subsequent events. If one wishes to prevent the manifestation of these demons, it is therefore not enough just to correct the first level, since an error in the second or third level of SA can lead to an error in a previous level.

These results are a first sign of a potential participation of SA demons in the type of accident under study here. We have been able to associate them with accident situations already known from the literature. In addition, SA demon sequences themselves occur in different patterns before the accident involving robotics occurs.

\subsection{SA demon patterns in industrial accidents}

Five SA demon appearance patterns were obtained at the end of our accident analysis. In particular, the Errant Mental Model appears in first position in all 5 patterns. In our results, other demons appear only if the Errant Mental Model appeared first. It can thus be considered as a demon which occurs systematically in this type of industrial accident. Consequently, this SA demon would be top of the priority list to address in order to prevent accidents involving robotics. Additionally, it is still important to note that it occurs, alone, in $13.33 \%$ of accidents.

The Out-of-the-loop SA demon was present in $86.67 \%$ of cases. This SA demon is particular in that it can appear at two distinct moments in accidents. Its appearance depends on how it is defined. For example, it may be linked with the Errant Mental Model. If the operator decides to enter the robot area thinking it to be safe, then it can be considered that he/she is already out-of-the-loop with the robot. On the other hand, it can be considered that the operator is out-of-the-loop if he/she is focused on his/her task and no longer realises that the robot is a nearby source of hazard. This second case could then concern patterns where the Attentional Tunneling SA demon appears. The latter is, in all cases, preceded by an Out-of-the-loop. These two possibilities are not, however, independent. Two patterns (40\% of the cases) included the SA demons 
Errant Mental Model, Out-of-the-loop and Attentional Tunneling. It is thus possible that the Out-of-the-loop SA demon manifested itself successively for Errant Mental Model and Attentional Tunneling. Finally Misplaced Salience can be considered as a demon preceding the accident. It occurs because the safety devices could not warn the operator about the possibility of an accident in the robot workspace.

It could be interesting to use these patterns also as an assessment criteria to analyse other accidents involving robotics. This would enable verifying whether they are systematic or context-dependent. After discussing the appearance patterns of SA demons in industrial accidents involving robotics, we directed our attention to their associations with the various possible interactions with humans in a robot workspace.

\subsection{SA demons in a robot workspace}

Our analysis allowed us to associate each demon with one of the interaction categories. Such associations could make it easier to target the elements of the robot workspace that can be studied in order to improve an operator's SA. In general, SA demons have been more often associated with errors in the human-robot $(46.29 \%)$ and humanenvironment $(42.29 \%)$ interaction categories. This is in line with our assumptions for the next steps of our project. Human-robot interactions being among the most problematic, it would then appear necessary to try to prevent the appearance of demons in this particular interaction category.

The Errant Mental Model SA demon has been associated mainly with errors in the human-robot and human-environment interactions categories. This is due to the fact that in many accident reports, the operator thought that the environment and the robot were safe. We found only one human-task interaction error for this demon. In this particular case, the victim thought his/her task could be carried out safely. It was in the context of that accident that the demon appeared in all three types of interaction at the same time. Thus, it demonstrated that the Errant Mental Model demon can be associated with all three interaction categories in a robot workspace and not just one. Based on the definition by Endsley and Jones (2016), this demon is part of the human mental model of the situation, which can therefore be altered for any of the elements of the robot workspace. A similar result has been found for the WAFOS demon. Indeed, this demon is also only linked to the mental state of the operator. Even though the robot can be a source of stress (Arai, Kato, and Fujita 2010), it is ultimately up to the operator to allow himself to be more or less disturbed by the robot.

The Out-of-the-loop SA demon has been associated only with the human-robot and human-environment interaction categories. The operator was not aware of the robot's motions and consequently of what was happening in the robot environment. Different results were obtained for the Misplaced Salience SA demon. This demon is mainly found in human-environment interactions and, to a lesser degree, in human-robot interactions. Indeed, the salient information about the state of the robot and the system in general can be found mainly in the "environment" part of the robotic system. This information is usually available on the command panels associated with the robot (Villani et al. 2018), and some information can be accessible directly on the robot (e.g. LEDs on a robot to inform about its status (Emika 2018)).

Finally, the Attentional Tunneling SA demon has only been found with errors in human-task interactions. Remember, this SA demon is where the operator is focused on a task and no longer pays attention to the other elements of the cell (i.e. the robot 
and the environment). In this way, the Tunneling of the operator is the root cause of the error in the context of the human-task interaction.

\subsection{Study limitations}

The first limitation of the proposed analysis is the paucity of information in the accident reports available in the EPICEA database. These reports depended on the accuracy of the description available to the reporters. In several instances, we were unable to identify SA demons, especially (WAFOS, Data Overload) that had been found in the analyses of numerous marine accidents (Stratmann and Boll 2016). With regard to the Complexity Creep and Requisite Memory Trap SA demons, as there is no, or very little, information about the procedures and the mental state of the operators, it was very difficult to determine their possible involvement in the reported accidents without constant monitoring of the operator's internal SA. In spite of this, we were still able to obtain promising results when it came to the demonstration of most SA demons encountered in the type of accident we studied here. An assessment criteria has been proposed for a systematic description of accidents in terms of SA degradation. Robotic accidents are analysed not only from an ergonomic point of view but also from a cognitive perspective in order to understand what led the victim to a degraded SA and finally to a human error and an accident.

Another limitation is linked to the very definition of SA demons and thus to their identification in each phase preceding the accident. Identification is sometimes based on subjective interpretations and personal appreciations of the situation. The subjectivity part is nevertheless mitigated by a consensus among all authors of this paper for the definitions of SA demons and additional elements for decision making.

\section{Conclusion}

Industrial accidents involving robotics have been analysed in detail to determine which factors can diminish an operator's SA. Specific SA demon patterns have been identified, as well as critical interactions.

Important concepts are involved - the robotic system and the robotic workspace, which include an operator, a robot, an environment and a task in an industrial context. Specific interaction issues have been recognised concerning human operators in interactions with other elements in the robot workspace. Next, two assessment criteria have been proposed to enable an analysis of accidents in the domain of industrial robotics. The first of these is inspired by work carried out in the field of aviation and degraded SA. There are eight factors called SA demons (Endsley and Jones 2016). The second is directly inspired by interactions with humans in the robot workspace. It allows the classification of SA demons into different interaction categories.

The accident analysis carried out during this study revealed the presence of five of the eight SA demons. It is possible to make a parallel with other reasons proposed in the literature to explain such accidents. SA demons led to or, at least, are involved in, the human errors which caused the accidents. Results showed that SA demons were mainly associated with the first level of SA (perception level). Another interesting finding is that SA demons have been generally classified in the human-robot and human-environment interaction categories. Several SA demons are very often grouped together (called patterns) to arrive at a degraded SA. Five typical patterns of SA demon occurrences have been identified. Statistically, it is not possible to infer any 
significant frequency but it would be interesting to investigate whether these any of these patterns could occur systematically in accidents involving industrial robotics.

\section{Recommendations}

Finally, recommendations can be drawn from the proposed analysis:

- This work has shown that operators have a degraded SA before accidents. Thus, SA demons are important factors that deserve to be taken into account when analysing potential human errors and determining risk issues.

- As many accidents involved an unexpected interaction between an operator and a robotic system, industrial robots may have to be designed such that interaction is made possible in certain circumstances, in total safety. Generally speaking, it is probably illusory to think that it is enough to forbid access to the robot area to avoid interactions and accidents. There will always be breakdowns, maintenance needs or special cases of use that will lead operators to interact with the robots. We therefore recommend to consider from the conception and design of the robot that there will necessarily be interactions, even if they are rare, and therefore to design cobotic systems rather than robotic-only systems, which implies to comply with complementary standards (ISO 2011b,a, 2016).

- It was repeatedly observed during the analysis that the operators were not aware of the state of the robot. In this vein, one solution might be to better inform the operator about it. It can be done in different ways: Thanks to a light, an alarm sound, a display panel, or by a residual movement of the robot, or several of these solutions at the same time. The choice of the best option is the subject of an ongoing study that we hope to publish soon.

\section{References}

Aghazadeh, Fereydoun, Robert Hirschfeld, and Robert Chapleski. 1993. "Industrial robot use: survey results and hazard analysis." In Proceedings of the Human Factors and Ergonomics Society Annual Meeting, Vol. 37-14, 994-998. SAGE Publications Sage CA: Los Angeles, CA.

Arai, Tamio, Ryu Kato, and Marina Fujita. 2010. "Assessment of operator stress induced by robot collaboration in assembly." CIRP annals 59 (1): 5-8.

Charpentier, P, and A Sghaier. 2012. "Industrial Robotic: Accident Analysis and Human-Robot Coactivity." In Proceedings of the "7th International Conference on the Safety of Industrial Automated Systems, SIAS, 11-12.

Chiappe, Dan L, Thomas Z Strybel, and Kim-Phuong L Vu. 2012. "Mechanisms for the acquisition of situation awareness in situated agents." Theoretical Issues in Ergonomics Science 13 (6): 625-647.

Colgate, J Edward, J Edward, Michael A Peshkin, and Witaya Wannasuphoprasit. 1996. "Cobots: Robots for collaboration with human operators." Proceedings of the 1996 ASME International Mechanical Engineering Congress and Exposition .

De Luca, Alessandro, and Fabrizio Flacco. 2012. "Integrated control for pHRI: Collision avoidance, detection, reaction and collaboration." In 2012 4th IEEE RAS 83 EMBS International Conference on Biomedical Robotics and Biomechatronics (BioRob), 288-295. IEEE.

Dhillon, BS. 1991. "Robot Reliability." In Robot Reliability and Safety, 119-149. Springer.

Emika, Franka. 2018. Panda. Technical Report. Accessed 2017-10-06.[Online]. Available: https://www. franka. de. 
Endsley, Mica R. 1988. "Design and evaluation for situation awareness enhancement." In Proceedings of the Human Factors Society annual meeting, Vol. 32-2, 97-101. SAGE Publications Sage CA: Los Angeles, CA.

Endsley, Mica R. 1995. "Toward a theory of situation awareness in dynamic systems." Human factors 37 (1): $32-64$.

Endsley, Mica R, and Debra G Jones. 2016. "SA demons: The enemies of situation awareness." In Designing for Situation Awareness, 50-61. CRC Press.

ISO. 2011a. 10218-2: 2011: Robots and robotic devices-Safety requirements for industrial robots-Part 2: Robot systems and integration. Standard ISO/IEC TR 10218-2:2011. Geneva, CH: International Organization for Standardization. https://www.iso.org/standard/41571.html.

ISO. 2011b. Robots and robotic devices-Safety requirements for industrial robots-Part 1: Robots. Standard ISO/IEC TR 10218-1:2011. Geneva, CH: International Organization for Standardization. https://www.iso.org/standard/51330.html.

ISO. 2012. Robots and robotic devices - Vocabulary. Standard ISO/IEC TR 8373:2012. Geneva, CH: International Organization for Standardization. https://www.iso.org/standard/55890.html.

ISO. 2016. TS 15066: 2016: Robots and robotic devices-Collaborative robots. Standard ISO/IEC TR 15066:2016. Geneva, CH: International Organization for Standardization. https://www.iso.org/standard/62996.html.

Jiang, Bernard C, and Charles A Gainer Jr. 1987. "A cause-and-effect analysis of robot accidents." Journal of Occupational accidents 9 (1): 27-45.

Jones, Debra G, and Mica R Endsley. 1996. "Sources of situation awareness errors in aviation." Aviation, space, and environmental medicine .

Karwowski, Waldemar, and Mansour Rahimi. 1991. "Worker selection of safe speed and idle condition in simulated monitoring of two industrial robots." Ergonomics 34 (5): 531-546.

Koppenborg, Markus, Peter Nickel, Birgit Naber, Andy Lungfiel, and Michael Huelke. 2017. "Effects of movement speed and predictability in human-robot collaboration." Human Factors and Ergonomics in Manufacturing \& Service Industries 27 (4): 197-209.

Lamy, P, and Claire Tissot. 2016. "Analyse de récits d'accidents du travail pour identifier des dérives d'usage et apport des TAL." Congrès Lambda Mu 20 de Maîtrise des Risques et de Sûreté de Fonctionnement, 11-13 Octobre 2016, Saint Malo, France.

Malm, Timo, Juhani Viitaniemi, Jyrki Latokartano, Salla Lind, Outi Venho-Ahonen, and Jari Schabel. 2010. "Safety of interactive robotics - learning from accidents." International Journal of Social Robotics 2 (3): 221-227.

Meziane, Ramy, Ping Li, Martin J-D Otis, Hassan Ezzaidi, and Philippe Cardou. 2014. "Safer hybrid workspace using human-robot interaction while sharing production activities." In 2014 IEEE International Symposium on Robotic and Sensors Environments (ROSE) Proceedings, 37-42. IEEE.

Michalos, George, Sotiris Makris, Panagiota Tsarouchi, Toni Guasch, Dimitris Kontovrakis, and George Chryssolouris. 2015. "Design considerations for safe human-robot collaborative workplaces." Procedia CIrP 37: 248-253.

Ogorodnikova, Olessia. 2008. "Human weaknesses and strengths in collaboration with robots." Periodica Polytechnica Mechanical Engineering 52 (1): 25-33.

Or, Calvin KL, Vincent G Duffy, and Chui Chui Cheung. 2009. "Perception of safe robot idle time in virtual reality and real industrial environments." International Journal of Industrial Ergonomics 39 (5): 807-812.

Rahimi, Mansour, and Waldemar Karwowski. 1990. "A research paradigm in human-robot interaction." International journal of industrial ergonomics 5 (1): 59-71.

Rasmussen, Jens. 1983. "Skills, rules, and knowledge; signals, signs, and symbols, and other distinctions in human performance models." IEEE transactions on systems, man, and cybernetics (3): 257-266.

Reason, James. 1990. Human error. Cambridge university press.

Salotti, Jean-Marc, Eric Ferreri, Olivier Ly, and David Daney. 2018. "Classification of Cobotic 
Systems." Ingénierie cognitique 1 (1). https://hal.archives-ouvertes.fr/hal-01943946.

Salotti, Jean-Marc, and Ephraim Suhir. 2019. "Degraded situation awareness risk assessment in the aerospace domain." In 2019 IEEE 5th International Workshop on Metrology for AeroSpace (MetroAeroSpace), 39-43. IEEE.

Schaub, Karlheinz, Gabriele Caragnano, Bernd Britzke, and Ralph Bruder. 2013. "The European assembly worksheet." Theoretical Issues in Ergonomics Science 14 (6): 616-639.

Shappell, Scott, Cristy Detwiler, Kali Holcomb, Carla Hackworth, Albert Boquet, and Douglas A Wiegmann. 2007. "Human error and commercial aviation accidents: an analysis using the human factors analysis and classification system." Human factors 49 (2): 227-242.

Stratmann, Tim Claudius, and Susanne Boll. 2016. "Demon hunt-the role of endsley's demons of situation awareness in maritime accidents." In Human-Centered and Error-Resilient Systems Development, 203-212. Springer.

Sugimoto, Nobaru, and Kunitomo Kawaguchi. 1983. "Fault tree analysis of hazards created by robots." In Proceedings of the 13th International Symposium on Industrial Robots and Robot, Vol. 7, 9-13.

Suhir, Ephraim, Sami Lini, Christophe Bey, Jean-Marc Salotti, Sylvain Hourlier, and Bernard Claverie. 2015. "Probabilistic modelling of the concept of anticipation in aviation." Theoretical Issues in Ergonomics Science 16 (1): 69-85.

Vasic, Milos, and Aude Billard. 2013. "Safety issues in human-robot interactions." In 2013 ieee international conference on robotics and automation, 197-204. IEEE.

Villani, Valeria, Fabio Pini, Francesco Leali, and Cristian Secchi. 2018. "Survey on humanrobot collaboration in industrial settings: Safety, intuitive interfaces and applications." Mechatronics 55: 248-266. 